\title{
The use of pharmacologic agents in the management of temporomandibular joint disorder
}

\author{
Chad Dammling $^{1}$, Shelly Abramowicz ${ }^{2}$, Brian Kinard ${ }^{1}$ \\ ${ }^{1}$ Department of Oral and Maxillofacial Surgery, School of Dentistry, University of Alabama at Birmingham, Birmingham, AL, USA; ${ }^{2}$ Division of \\ Oral and Maxillofacial Surgery, Department of Surgery, Emory University School of Medicine, Chief, Oral and Maxillofacial Surgery, Children's \\ Healthcare of Atlanta, Atlanta, GA, USA \\ Contributions: (I) Conception and design: All authors; (II) Administrative support: All authors; (III) Provision of study materials or patients: All \\ authors; (IV) Collection and assembly of data: All authors; (V) Data analysis and interpretation: All authors; (VI) Manuscript writing: All authors; (VII) \\ Final approval of manuscript: All authors. \\ Correspondence to: Shelly Abramowicz, DMD, MPH, FACS. Oral and Maxillofacial Surgery, Emory University, 1365 Clifton Road, Bldg B, Suite \\ 2300, Atlanta, GA 30322, USA. Email: sabram5@emory.edu.
}

\begin{abstract}
Temporomandibular joint disorders (TMD) are oro-facial pain conditions that originate from either intraarticular or extraarticular related pathology. Following an accurate diagnosis, there are a variety of non-surgical and surgical management options available. The aim of this article is to review the available pharmacologic agents for the management of extraarticular and intraarticular TMD. These medical options are often first line and are combined with other non-surgical modalities. There are multiple pharmacologic options utilized to treat TMD, from non-steroidal anti-inflammatory drugs (NSAIDs) to muscle relaxants and steroids. Many of these medications are used synergistically to provide symptom improvement and prevention of persistent disease. This paper will discuss the use of the following classes of medications used to manage TMD: NSAIDs, corticosteroids, narcotics, muscle relaxants, anticonvulsants, anxiolytics, and topical therapy. Despite their extensive clinical use, there remains insufficient evidence to recommend one therapy over another. This is due to the lack of systematic reviews and meta-analyses in the current literature. For this reason, there remains a need for a randomized control trial with clear pre-pharmacotherapy diagnoses, blinding, and research objectives. NSAIDs have been recommended as first line therapy for intraarticular disorders with the addition of muscle relaxants if there is a muscle component. Several of the other medications discussed are often patient specific or given secondarily when previous therapy has failed. It is critical to recognize systemic patient factors when prescribing any of these medications to avoid side effects and drug-drug interactions.
\end{abstract}

Keywords: Temporomandibular joint disorder (TMD); medical therapy; non-surgical management

Received: 22 June 2020; Accepted: 31 May 2021; Published: 10 June 2022.

doi: 10.21037/fomm-20-37

View this article at: http://dx.doi.org/10.21037/fomm-20-37

\section{Introduction}

Temporomandibular joint disorders (TMD) encompass a wide variety of diseases that affect the temporomandibular joint itself (intraarticular) and/or the surrounding musculature (extraarticular) (1-3). Some of the studied etiologic factors associated with TMD include a history of trauma, systemic related inflammatory conditions, and psychosocial issues (4). Symptoms consist of pain, joint sounds, limited mouth opening, and/or tenderness in the preauricular region (5). These conditions affect females more frequently than males and are most common between the ages of 20-40 (5). TMD has been estimated to affect between $3-12 \%$ of the general population (6).

As with any disease, an accurate diagnosis must be made prior to treatment. The Diagnostic Criteria for TMD 
(DC/TMD) was created to standardize diagnoses based on epidemiologic studies from multiple centers $(6,7)$. The DC/TMD provides a standard for diagnosis and evaluates both physical signs and symptoms (Axis I) with psychosocial factors (Axis II) $(5,7,8)$. It is critical that a thorough systemic evaluation be completed prior to diagnosis as several psychosocial disorders can affect any therapeutic intervention (2).

Utilizing these DC/TMJ criteria, the practitioner can determine if the etiology of the patient's symptoms originates from an intraarticular or an extraarticular issue. Finding an intraarticular condition, the management goal should be aimed at decreasing joint related function. If symptoms are diagnosed as extraarticular and related to the surrounding musculature/soft tissues, management goals are then directed to decreasing any masticatory and/or cervical muscular hyperactivity with associated tissue inflammation. Although pharmacotherapy is a first line option, it is rarely used alone, and is often combined with other non-surgical modalities (9). It is critical that the patient is aware that any management option for TMD is not meant to be a cure, but rather an aid to improve mandibular function, relief of pain, increase quality of life, and prevent further progression of the disease (10).

\section{Nonsteroidal anti-inflammatory drugs (NSAIDs)}

NSAIDs block arachidonic acid conversion into prostaglandins through the inhibition of cyclooxygenase enzymes (COX-1 and COX-2). COX-1 is a constitutive enzyme that plays a critical role in normal homeostatic functions including platelet aggregation, renal blood flow, and gastric mucosa protection. COX-2 is an induced enzyme that is stimulated by inflammatory mediators to promote inflammation and the formation of prostaglandins that mediate swelling, pain, and fever. All NSAIDs are effective in blocking both COX-1 and COX- 2 but there is a varying degree to which NSAID acts on which enzyme (1). NSAIDs can generally be classified as non-selective (both COX-1 and COX-2 inhibition) or selective (COX-2) inhibitors. The inhibition of cyclooxygenase is normally reversible with the exception of aspirin, where the enzyme is acetylated and irreversibly inhibited (1).

Nonsteroidal anti-inflammatory drugs aid in the management of TMD through their anti-inflammatory and analgesic properties. These medications decrease the release of local inflammatory mediators thereby decreasing inflammation within the joint and associated muscles and soft tissues $(4,7)$.

NSAIDs have been shown to be beneficial in numerous other inflammatory conditions including osteoarthritis and synovitis (1). It is critical to keep in mind that certain NSAIDs have side effects including gastrointestinal irritation, increased bleeding, and nephrotoxicity. The risk of several of these side effects can be greatly increased by concomitant ingestion of other medications. GI irritation and potential bleeding has been shown to increase when the patient is also taking selective serotonin receptor inhibitors, alcohol, or herbal medications such as gingko biloba or St. John's wort. Although selective COX-2 inhibitors (e.g., celecoxib) have decreased gastrointestinal side effects, consistent use has been shown to increase cardiovascular events, especially in those with cardiac risk factors (10).

NSAIDs may also affect the metabolism of other medications. Several non-steroidal medications have been reported to decrease clearance of specific medications such as methotrexate and lithium leading to potentially toxic levels of these drugs. Several antihypertensive medications are also affected by renal prostaglandins (diuretics and ACE inhibitors) and can therefore have reduced efficacy with the use of an NSAID (11). A secondary concern with combining the use of NSAIDs with ACE inhibitors is that there is an increased risk of kidney damage given that both medications reduce blood flow to the kidney (11).

Several systematic reviews analyzed NSAIDs for the management of TMD pain, but none have reached a definitive, quantitative conclusion on their efficacy. This is due to a combination of factors such as nonspecific initial diagnoses, variable control groups, and diverse choices in protocols for dosage (12). To circumvent this problem, Kulkarni et al. attempted to adjust diagnoses in prior studies to match the DC/TMD criteria. In their systematic review, despite this adjustment, the variance in diagnoses remained too heterogenous to determine the efficacy of NSAIDs on TMD pain (12).

Even with these inconclusive findings, NSAIDs remain first line therapy for TMD pain. They are considered safe and effective when utilized for other chronic and acute inflammatory conditions. Several weeks of consistent NSAID therapy is often indicated to obtain maximal effects of these medications (3). One of the most commonly prescribed NSAIDs for TMD treatment is naproxen $\left(\right.$ Aleve $\left.^{\circledR}\right)$. In a prospective, six-week, double-blind study, patients were placed into three parallel groups: naproxen $500 \mathrm{mg}$ twice daily, celecoxib $100 \mathrm{mg}$ twice daily, or a placebo. Naproxen was found to significantly decrease 
TMD pain and improve mandibular range of motion compared to celecoxib and the placebo $(13,14)$. Both NSAID medications were well tolerated, but celecoxib was found to have no significant effect on symptoms compared to placebo $(2,4,5)$.

\section{Corticosteroids}

In contrast to NSAIDs, corticosteroids disrupt the inflammatory pathway by blocking arachidonic acid production through the inhibition of the enzyme phospholipase. This inhibition occurs earlier on in the inflammatory cascade and results in a greater effect on inflammatory mediators than NSAIDs (1). In addition to this blockade, corticosteroids cause a decrease in the number of proinflammatory cells such as lymphocytes and macrophages (10).

The side effects of systemic corticosteroids must be taken into consideration prior to their use, even when considering short term use (15). Low dose prednisone (10-20 mg daily) for over two weeks can suppress the hypothalamic-pituitaryadrenal axis. Evaluation of long-term therapy is especially significant as it can result in a Cushingoid disease process, osteoporosis, electrolyte instability, hypotension, diabetes, and acute adrenal crisis (15). Therefore, if indicated for acute treatment, the dose should be tapered over 5-7 days.

Corticosteroids are sometimes instilled directly into the TMJ. This approach is discussed elsewhere in this series. For oral administration, short term steroids should be reserved for patients with severe joint inflammation related to severe osteoarthritis or rheumatoid arthritis (10).

\section{Narcotic}

Narcotic analgesics are centrally acting opioids used to alleviate severe pain. Three major opioid receptors exist including mu, kappa, and delta. The primary analgesic effects of opiates act through the $m u$ receptor which also leads to several side effects.

Given the high risk of dependence while using opiates, it is critical to educate patients of addiction potential and provide only short term prescriptions for acute pain control (1). The most commonly prescribed opiates for acute TMJ pain are codeine, hydrocodone, or oxycodone (4). Tolerance to these medications can lead to higher dosage requirements to maintain analgesic activity. The most common side effects of opiates are respiratory depression, sedation, constipation, nausea, and vomiting. Narcotics are generally not considered to be a first line therapy for TMD $(4,10)$.

Tramadol is an additional opiate-like drug that has been shown efficacy in the treatment of chronic pain symptoms with fibromyalgia, lower back pain, and osteoarthritis (10). In these conditions, tramadol, or combination tramadol with acetaminophen, provided improvement in symptoms. Although tramadol only partially binds to opioid $m u$ receptors, it still must be used with caution in long-term therapy situations. Additionally, tramadol should not be used in patients on monoamine oxidase inhibitors (MAOIs) due to risk of developing serotonin syndrome.

\section{Muscle relaxants}

Muscle relaxants are commonly utilized in the management of TMD and are helpful in decreasing hyperactivity associated with excessive masticatory and cervical muscle contraction. Muscle relaxants are classified as centrally or peripherally acting. Centrally acting muscle relaxants are more commonly prescribed for the management of muscle related TMD symptoms. The most commonly used centrally acting muscle relaxants are methocarbamol and cyclobenzaprine (1). Peripherally acting muscle relaxants include baclofen and botulinum toxin $\mathrm{A}$.

Centrally acting muscle relaxants decrease neural output to voluntary muscles involving the brainstem, thalamus, and basal ganglia. They are recommended to be taken at night to circumvent the common side effects of drowsiness and fatigue.

Cyclobenzaprine has been found to provide symptomatic muscle related pain relief and decrease muscular spasm in the cervical and lumbar regions compared to placebo (3). Additionally, systematic reviews have shown that cyclobenzaprine has a beneficial effect specifically on TMD muscle related pain (14). A three-week randomized controlled trial by Herman et al. reported nightly cyclobenzaprine $10 \mathrm{mg}$ was superior to placebo or clonazepam $0.5 \mathrm{mg}$ in decreasing jaw pain upon wakening (10). These patients were also provided written and verbal information on the management of TMJ pain including education on the pathophysiology of TMD, "pain free diets", and monitoring sleeping positions thus enforcing the need for comprehensive therapy.

Despite these positive findings, many of the muscle relaxant studies have limited follow up and long-term data on their efficacy is minimal (14). Unlike other medications in this class, cyclobenzaprine's chemical structure also 
closely resembles that of tricyclic antidepressants (TCAs). For this reason, anticholinergic side effects (e.g., xerostomia, tachycardia, etc.) must be monitored and this medication must not be prescribed to patients taking MAOIs.

\section{Anticonvulsants}

Anticonvulsant medications are commonly used to manage neuropathic pain and therefore, play a potential role in the management of TMD. These agents inhibit neuronal excitation at several sites: voltage gated calcium channels, glutamate receptors, N-methyl-D-aspartate (NMDA) receptors, and gamma-aminobutyric acid (GABA) receptors (6). Both gabapentin and pregabalin have been used in the management of TMD and other chronic pain syndromes although their exact mechanism for analgesia remains unclear (3). The most frequent adverse side effects are dizziness, somnolence, and peripheral edema (3).

Gabapentin has been reported to reduce spontaneous TMD myogenous pain and tender trigger sites at the temporalis and masseter compared to placebo $(10,16)$. In this randomized controlled trial, the initial dose of gabapentin was $300 \mathrm{mg}$. The dose was titrated up every three days until pain was relieved. Spontaneous pain reduction occurred by week eight and a decrease in muscle related point tenderness was noted at week twelve. Although helpful for myogenous pain, these medications are rarely a first line and are indicated for chronic pain patients who have a history of failed TMJ surgeries, or have had longstanding centrally mediated pain symptoms (10). Prescribing these medications for TMD is restricted to physicians that have familiarity with the side effect profile and drug-drug interactions.

\section{Antidepressants}

There are several classes of antidepressants that have been used in the treatment of TMD: SSRIs, serotonin and norepinephrine reuptake inhibitors (SNRIs), TCAs, and MAOIs. As previously discussed, it is critical to determine if a patient with TMD symptoms has a coexisting psychological disorder, as this can affect their long-term management. There is a correlation between chronic pain symptoms and depression which must be considered in order to provide appropriate and comprehensive patient care (1).

The most common antidepressant used to treat TMD symptoms are TCAs. TCAs have analgesic properties and proven efficacy. Amitriptyline has specifically been shown to have analgesic properties at low doses and demonstrated efficacy in management of TMD (17). In placebo controlled trials of post-herpetic neuralgia and chronic lower back pain, amitriptyline and imipramine were shown to be effective for reducing pain in depressed and non-depressed patients (10). In addition, TCAs have been shown to aid in other chronic pain states such as migraines, tension headaches, cancer, and arthritis (18). For patients with a history of chronic TMJ pain, two weeks of low dose amitriptyline $(25 \mathrm{mg} /$ day $)$ was significantly more effective than placebo in reducing pain and discomfort as measured via visual analog scale (17). Another study showed that low dose amitriptyline (10-30 mg/day) provided improvement in pain at six weeks and one year of treatment (10). No greater pain relief was found when amitriptyline was prescribed in higher dosages (50-75 mg/day) (18). Side effects of TCAs are sedation, xerostomia, and cardiac complications. Similar to many of the medications previously discussed, additional studies with larger patient samples and definitive diagnostic criteria will help determine the efficacy of TCAs in TMD pain (18).

SSRIs are also effective in treating TMD. These medications lead to increased synaptic serotonin by blocking neuronal transport (3). Citalopram and paroxetine specifically have been shown to relieve neuropathic pain symptoms and aid in easing bruxism $(3,7)$. Additionally, the SNRI duloxetine has shown efficacy in the management of diabetic neuropathy, fibromyalgia, and chronic muscular pain. For this reason, it is theorized that SNRIs also have a role in the management of myofascial pain (10).

It is important to note that prescribing antidepressant medications is limited to clinicians with experience treating chronic pain. There are several side effects and preoperative evaluations that must be completed prior to initiating this therapy. Additionally, off-label dosage for chronic pain is significantly less than the medication regimen for depression.

\section{Anxiolytics}

Although less frequently used in the management of TMD, anxiolytics have been shown to decrease masticatory muscle hyperactivity and nocturnal bruxism. The most commonly used anxiolytics are benzodiazepines- specifically diazepam or clonazepam because they have anticonvulsant activity and long half-lives. Benzodiazepines act by binding receptors in the central nervous system and increase the 
efficiency of gamma aminobutyric acid (GABA) allowing for hyperpolarization of the cell. This mechanism contributes to the anxiolytic and sedative properties of the medication (10).

Similar to opiates, anxiolytics medications can cause psychomotor impairment and dependence; long-term use is contraindicated. Dosages must be altered in the elderly since they have a decrease in clearance and an increase in sensitivity (10). Sedation and confusion are the most common side effects associated with these medications. Generally, benzodiazepines are utilized at bedtime in patients with hyperactive muscle symptoms in order to reduce excessive muscle contraction overnight (10).

In one double blind, placebo-controlled study, patients dosed with diazepam $5 \mathrm{mg}$ four times per day for four weeks had improvement in muscle related pain symptoms compared to placebo. In this same study, those dosed with ibuprofen $600 \mathrm{mg}$ every six hours had no benefit unless diazepam was also added $(10,19)$. In another study comparing the effects of diazepam, piroxicam, and a placebo, diazepam was shown to significantly improve TMD muscle related pain symptoms over two weeks (20). Clonazepam has also been shown to significantly help TMD muscle related symptoms in patients that failed oral appliance and physical therapy (21). Regardless of these beneficial outcomes, benzodiazepines are rarely utilized in the management of muscle related TMD due to adverse side effects, tolerance, and dependence (3).

\section{Topical therapy}

Topical use of NSAIDs through gels or ointments has been shown to be effective. This method requires less long-term use and has less side effects $(12,22)$. Topical diclofenac gel has been reported to achieve concentrations high enough to inhibit prostaglandin E2 and to decrease TMJ nociceptor transmission. Use of this topical agent four times per day (15 mg/mL) for two weeks was found to be as effective as oral diclofenac (50 $\mathrm{mg}$ twice daily) for TMD muscle related pain $(10,23)$. Despite these improvements in muscle related symptoms, available studies lacked a definitive TMD diagnosis and were of a small sample size. As a result, it is difficult to determine the true efficacy of topical therapy (22).

Capsaicin and lidocaine have also been utilized topically for the relief of TMD muscle related pain. Capsaicin is a derivative of chili pepper and has been shown to help manage osteoarthritis and neuropathic muscle related pain. This cream depletes substance $\mathrm{P}$ and inflammatory mediators from nerve endings (10). The cream can be utilized in $0.025 \%$ to $0.075 \%$ and can often cause a burning sensation on initial application. In a randomized, double-blind, placebo-controlled study on thirty patients evaluating the use of capsaicin cream, there was significant improvement in pain to muscle palpation and subjective pain relief in both the treatment and placebo groups after four weeks (24). For this reason, the authors hypothesized that any topical therapy can possibly aid in symptom improvement due to the placebo effect.

Lidocaine transdermal patches have been FDA approved for the management of postherpetic neuralgia and have been efficacious in the management of chronic lower back pain and osteoarthritis muscle related pain. The patch can be cut into a variety of smaller sizes for use and applied for approximately twelve hours. Given its efficacy in other chronic pain conditions, it is theorized that lidocaine patch therapy could also improve TMD muscle related pain.

\section{Summary}

There are a variety of pharmacologic strategies that can be utilized in the management of TMD. Medication therapy is rarely utilized alone and is often used in conjunction with other modalities such as oral appliances and/or physical therapy. It is important the patient is aware that medical management provides improvement of symptoms, but not a cure (10). Unfortunately, despite longstanding use of these therapies, there remains limited data to recommend one therapy over another.

In a 2010 Cochrane review article, it was noted that there is insufficient evidence regarding the effectiveness of medical management for TMD (25). In this systematic review, eleven studies were included, however, a majority were excluded due to lack of a placebo group, insufficient reporting, and no clear diagnosis of TMD. Several other systematic reviews, specific to pharmacologic interventions for TMD, report similar problems in study design leading to unclear conclusions regarding medication use $(12,18,22)$. For this reason, there is a need for well-conducted randomized control trials in the management of TMD with clear research objectives (25).

Despite this lack of evidence, NSAIDs (i.e., naproxen ${ }^{\circledR}$ ) have generally remained the first line medication for patients with significant inflammatory pain. For patients that have a muscular component to their pain, muscle relaxants have been recommended in conjunction with NSAIDs. Cyclobenzaprine has a beneficial effect on TMD pain when symptoms are muscle related. Many other 
therapies (e.g., TCAs, benzodiazepines, anticonvulsants, and topical therapy) can be considered for individual cases to match the patient's symptoms and medical comorbidities. The use of these secondary medications including TCAs and anticonvulsants is generally restricted to clinicians with experience in chronic pain management.

Additionally, it is critical to discuss with the patient the dose and length of management prior to initiating therapy. Providing "as needed" medications can lead to a cyclical process of symptoms which can further complicate management. For these reasons, a strict time contingent dosage schedule is more appropriate in order to break the cyclic symptoms associated with the disease process along with establishment of an end point for reevaluation (10). Further, given the multiple medical comorbidities or medications that these patients may have, it is imperative to recognize any drug interactions or contraindications prior to prescribing.

\section{Acknowledgments}

Funding: None.

\section{Footnote}

Provenance and Peer Review: This article was commissioned by the Guest Editors (Stephen Feinberg and Louis Mercuri) for the series "Temporomandibular Joint Disorders Diagnosis and Management-What Does the Future Hold?" published in Frontiers of Oral and Maxillofacial Medicine. The article has undergone external peer review.

Conflicts of Interest: The authors have completed the ICMJE uniform disclosure form (available at https://fomm. amegroups.com/article/view/10.21037/fomm-20-37/coif). The series "Temporomandibular Joint Disorders Diagnosis and Management-What Does the Future Hold?" was commissioned by the editorial office without any funding or sponsorship. The authors have no other conflicts of interest to declare.

Ethical Statement: The authors are accountable for all aspects of the work in ensuring that questions related to the accuracy or integrity of any part of the work are appropriately investigated and resolved.

Open Access Statement: This is an Open Access article distributed in accordance with the Creative Commons
Attribution-NonCommercial-NoDerivs 4.0 International License (CC BY-NC-ND 4.0), which permits the noncommercial replication and distribution of the article with the strict proviso that no changes or edits are made and the original work is properly cited (including links to both the formal publication through the relevant DOI and the license). See: https://creativecommons.org/licenses/by-nc-nd/4.0/.

\section{References}

1. Indresano AT, Park CM. 39 - Nonsurgical Management of Temporomandibular Joint Disorders [Internet]. Third Edit. Oral and Maxillofacial Surgery. Elsevier Inc., 2020:865-82. Available online: http://dx.doi.org/10.1016/ B978-0-323-41499-9.00082-0

2. Gil-Martínez A, Paris-Alemany A, López-de-UraldeVillanueva I, et al. Management of pain in patients with temporomandibular disorder (TMD): Challenges and solutions. J Pain Res 2018;11:571-87.

3. Ouanounou A, Goldberg M, Haas D. Pharmacotherapy in Temporomandibular Disorders: A Review. J Can Dent Assoc 2017;83:h7-8.

4. Dym H, Bowler D, Zeidan J. Pharmacologic Treatment for Temporomandibular Disorders. Dent Clin North Am 2016;60:367-79.

5. Wieckiewicz M, Boening K, Wiland P, et al. Reported concepts for the treatment modalities and pain management of temporomandibular disorders. J Headache Pain 2015;16:106-12.

6. Heir GM. The Efficacy of Pharmacologic Treatment of Temporomandibular Disorders. Oral Maxillofac Surg Clin North Am 2018;30:279-85.

7. Abouelhuda AM, Khalifa AK, Kim YK, et al. Non-invasive different modalities of treatment for temporomandibular disorders: Review of literature. J Korean Assoc Oral Maxillofac Surg 2018;44:43-51.

8. Schiffman E, Ohrbach R, Truelove E, et al. Diagnostic Criteria for Temporomandibular Disorders (DC/TMD) for Clinical and Research Applications: Recommendations of the International RDC/TMD Consortium Network* and Orofacial Pain Special Interest Group. J Oral Facial Pain Headache 2014;28:6-27.

9. Dionne RA. Pharmacologic treatments for temporomandibular disorders. Oral Surg Oral Med Oral Pathol Oral Radiol Endod 1997;83:134-42.

10. Hersh E V, Balasubramaniam R, Pinto A. Pharmacologic Management of Temporomandibular Disorders. Oral Maxillofac Surg Clin North Am 2008;20:197-210. 
11. Moore N, Pollack C, Butkerait P. Adverse drug reactions and drug-drug interactions with over-the-counter NSAIDs. Ther Clin Risk Manag 2015;11:1061-75.

12. Kulkarni S, Thambar S, Arora H. Evaluating the effectiveness of nonsteroidal anti-inflammatory drug(s) for relief of pain associated with temporomandibular joint disorders: A systematic review. Clin Exp Dent Res 2020;6:134-46.

13. Ta LE, Dionne RA. Treatment of painful temporomandibular joints with a cyclooxygenase- 2 inhibitor: A randomized placebo-controlled comparison of celecoxib to naproxen. Pain 2004;111:13-21.

14. Häggman-Henrikson B, Alstergren P, Davidson T, et al. Pharmacological treatment of oro-facial pain - health technology assessment including a systematic review with network meta-analysis. J Oral Rehabil 2017;44:800-26.

15. Grennan D, Wang S. Steroid Side Effects. JAMA 2019;322:282.

16. Kimos P, Biggs C, Mah J. Analgesic action of gabapentin on chronic pain in the masticatory muscles: a randomized controlled trial. Pain 2007;127:151-60.

17. Rizzatti-Barbosa CM, Nogueira MTP, de Andrade ED, et al. Clinical Evaluation of Amitriptyline for the Control of Chronic Pain Caused by Temporomandibular Joint Disorders. Cranio 2003;21:221-5.

18. Cascos-Romero J, Vázquez-Delgado E, VázquezRodríguez E, et al. The use of tricyclic antidepressants in the treatment of temporomandibular joint disorders: Systematic review of the literature of the last 20 years.

\section{doi: $10.21037 /$ fomm-20-37}

Cite this article as: Dammling C, Abramowicz S, Kinard B. The use of pharmacologic agents in the management of temporomandibular joint disorder. Front Oral Maxillofac Med 2022;4:17.
Med Oral Patol Oral Cir Bucal 2009;14:E3-7.

19. Singer E, Dionne R. A Controlled Evaluation of Ibuprofen and Diazepam for Chronic Orofacial Muscle Pain. J Orofac Pain 1997;11:139-46.

20. Roldan OV, Maglione H, Mainieri S, et al. Carreiri R. Piroxicam, diazepam and placebo in the treatment of temporomandibular joint dysfunction. Double blind study. Rev Asoc Odontol Argent 1990;78:83-5.

21. Harkins S, Linford J, Cohen J, et al. Administration of clonazepam in the treatment of TMD and associated myofascial pain: a double-blind pilot study. J Craniomandib Disord 1991;5:179-86.

22. Senye M, Mir CF, Morton S, et al. Topical nonsteroidal anti-inflammatory medications for treatment of temporomandibular joint degenerative pain: a systematic review. J Orofac Pain 2012;26:26-32.

23. Di Rienzo Businco L, Di Rienzo Businco A. Topical versus systemic diclofenac in the treatment of temporomandibular joint dysfunction symptoms. Acta Otorhinolaryngol Ital 2004;24:279-83.

24. Winocur E, Gavish E, Halachmi M, et al. Topical Application of Capsaicin for the Treatment of Localized Pain in the Temporomandibular Joint Area. J Orofac Pain 2000;14:31-6.

25. Mujakperuo HR, Watson M, Morrison R, et al. Pharmacological interventions for pain in patients with temporomandibular disorders. Cochrane Database Syst Rev 2010;(10):CD004715. 